\title{
ON THE GEOMETRY OF THE PAINLEVÉ V EQUATION AND A BÄCKLUND TRANSFORMATION
}

\author{
W. K. SCHIEF ${ }^{1}$
}

(Received I May, 2000)

\begin{abstract}
It is shown that an integrable class of helicoidal surfaces in Euclidean space $\mathbb{E}^{3}$ is governed by the Painlevé $V$ equation with four arbitrary parameters. A connection with sphere congruences is exploited to construct in a purely geometric manner an associated Bäcklund transformation.
\end{abstract}

\section{Introduction}

It has been demonstrated in $[8]$ that the integrable generalized Ernst equation $[1,10]$

$$
E_{z \bar{z}}+\frac{1}{2} \frac{p_{\bar{z}} E_{z}+p_{z} E_{\bar{z}}}{\Re(p)}=\frac{E_{z} E_{\bar{z}}}{\Re(E)}, \quad p_{z \bar{z}}=0
$$

which governs the interaction of 'neutrino' and gravitational fields in axially symmetric space-times of general relativity, admits Lie-point symmetry reductions to the Painlevé III, V and VI equations with arbitrary parameters. The above 'Ernst-Weyl' equation has also been identified [7] as a canonical 2+0-dimensional reduction of the 2+1dimensional Loewner-Konopelchenko-Rogers (LKR) integrable system [5]. This connection has been exploited in [9] to construct a Laplace-Darboux-type invariance of the nonlinear Ernst-Weyl equation. Indeed, if $(E, p)$ is a solution of the Ernst-Weyl equation (1) then the Laplace-Darboux-type transforms

$$
\mathrm{E}_{ \pm}=\mathscr{L}_{ \pm}(\mathrm{E}), \quad \mathrm{p}_{ \pm}=\mathscr{L}_{ \pm}(\mathrm{p})
$$

\footnotetext{
'School of Mathematics, The University of New South Wales, Sydney, NSW 2052, Australia. (C) Australian Mathematical Society 2002, Serial-fee code 1446-181 1/02
} 
defined by

with

$$
\begin{aligned}
& E_{+}=\frac{E \Re(E) p_{z}+\Re(p) \bar{E}_{z}}{\Re(E) p_{z}-\mathfrak{R}(p) E_{z}}, \quad p_{+}=p-2 i v, \\
& E_{-}=\frac{E \Re(E) p_{\bar{z}}+\Re(p) \bar{E}_{\bar{z}}}{\Re(E) p_{\bar{z}}-\mathfrak{R}(p) E_{\bar{z}}}, \quad p_{-}=p+2 i v,
\end{aligned}
$$

$$
\nu_{z}=\mathrm{i} \rho_{z}, \quad \nu_{\bar{z}}=-\mathrm{i} \rho_{\bar{z}}, \quad \rho=\Re(\mathrm{p})
$$

constitute another two solutions of the Ernst-Weyl equation. In particular, if $\Im(p)=0$ mod $2 v$ then the Ernst-Weyl equation may be reduced to the Ernst equation corresponding to $\Im(p)=0$ by means of iterative application of the Laplace-Darboux-type transformation $\mathscr{L}_{+}$or its inverse $\mathscr{L}_{-}=\mathscr{L}_{+}^{-1}$.

The Ernst-Weyl equation (1) may be shown to appear in connection with a novel class of integrable surfaces ('generalized Weingarten surfaces') in Minkowski space $\mathbb{M}^{3}$ which was introduced in [6]. The solutions of the Ernst-Weyl equation in terms of the Painlevé III, V and VI transcendents may therefore be interpreted geometrically. Furthermore, the above Laplace-Darboux-type transformations admit a simple geometric interpretation in terms of sphere congruences so that their action on the Painlevé equations may also be placed on a geometric basis.

For brevity and simplicity, we here focus on the $O(3)$ analogue of the Ernst-Weyl equation which is descriptive of the spherical representation of generalized Weingarten surfaces of Class 2 in Euclidean space $\mathbb{E}^{3}$ and show how the Painlevé V equation is obtained as a particular reduction by consideration of helicoidal surfaces. It is demonstrated that the Laplace-Darboux-type transformation for generalized Weingarten surfaces of Class 2 set down in [6] is compatible with the reduction to the Painlevé V equation and therefore induces an associated Bäcklund transformation.

\section{Generalized Weingarten surfaces}

In the following, we are concerned with the geometry of surfaces in Euclidean space $\mathbb{E}^{3}$. Thus if the position vector of a surface $\Sigma$ is denoted by $r$ then the surface is determined up to its position in space by the fundamental forms [3]

$$
\mathrm{I}=d \boldsymbol{r} \cdot d \boldsymbol{r}, \quad \mathrm{II}=-d \boldsymbol{r} \cdot d \boldsymbol{N},
$$

where $\boldsymbol{N}$ designates the unit normal to the surface. The third fundamental form

$$
\mathrm{III}=d N \cdot d N,
$$


which constitutes the quadric form of the spherical representation of $\Sigma$, is related to the first and second fundamental forms by

$$
\mathscr{K} \mathrm{I}-\mathscr{M} \mathrm{II}+\mathrm{III}=0 .
$$

Here $\mathscr{K}$ and $\mathscr{M}$ denote the Gaußian and mean curvatures respectively. The class of integrable surfaces which is relevant in the present context has been introduced in [6].

DEFINITION 2.1 (Generalized Weingarten surfaces of Class 2). A surface $\Sigma \subset \mathbb{E}^{3}$ is said to be a generalized Weingarten surface of Class 2 if there exist two functions $\mu$ and $\rho$ which are harmonic with respect to the quadratic form

$$
\mathrm{II}+\mu \mathrm{III}
$$

and the relation

$$
\left(\mu^{2}-\rho^{2}\right) \mathscr{K}+\mu \mathscr{M}+1=0
$$

is satisfied.

Since the functions $\mu$ and $\rho$ are harmonic, it is natural to introduce conformal coordinates with respect to (5), that is, complex coordinates $z, \bar{z}$ are chosen such that

$$
\mathrm{II}+\mu \mathrm{III} \sim d z d \bar{z} .
$$

Hence, generalized Weingarten surfaces of Class 2 admit the following canonical parametrization [6]:

THEOREM 2.2 (Parametrized generalized Weingarten surfaces of Class 2). Generalized Weingarten surfaces of Class 2 may be parametrized in such a way that the generalized Lelieuvre formulae

$$
r_{z}=\mathrm{i} \rho N_{z} \times N+\mu N_{z}, \quad r_{\bar{z}}=\mathrm{i} \rho N \times N_{\bar{z}}+\mu N_{\bar{z}}
$$

hold and

$$
\rho_{z \bar{z}}=0, \quad \mu_{z \bar{z}}=0 .
$$

If the functions $\mu$ and $\rho$ are constant then Definition 2.1 reduces to that for 'linear' Weingarten surfaces [3]. If $\mu=0$ then $\rho$ is harmonic with respect to the second fundamental form and $(1 / \sqrt{\mathscr{K}})_{z \bar{z}}=0$ so that Bianchi surfaces of positive Gaußian curvature are obtained [2]. In the case $\mu= \pm \rho, \rho$ is harmonic with respect to the first fundamental form by virtue of (4) and $(1 / \mathscr{M})_{z \bar{z}}=0$. This corresponds to the definition of harmonic inverse mean curvature surfaces [2].

The compatibility condition $\boldsymbol{r}_{z \bar{z}}=\boldsymbol{r}_{\bar{z} z}$ for the generalized Lelieuvre formulae (6) can be conveniently expressed in terms of a complex function $E$ ('Ernst potential') 
which labels the complex plane onto which the unit normal $N$ is stereographically projected via

$$
N=\frac{1}{|E|^{2}+1}\left(\begin{array}{c}
E+\bar{E} \\
-\mathrm{i}(E-\bar{E}) \\
|E|^{2}-1
\end{array}\right) .
$$

Thus, if one introduces a complex harmonic function $p$ according to

$$
\mathrm{P}=\rho+\mathrm{i} \sigma, \quad \sigma_{z}=-\mathrm{i} \mu_{z}, \quad \sigma_{\bar{z}}=\mathrm{i} \mu_{\bar{z}},
$$

then any generalized Weingarten surface of Class 2 gives rise to a solution of the Ernst-type equation

$$
\mathrm{E}_{z \bar{z}}+\frac{1}{2} \frac{\mathrm{p}_{\bar{z}} \mathrm{E}_{z}+\mathrm{p}_{z} \mathrm{E}_{\bar{z}}}{\mathfrak{R}(\mathrm{p})}=2 \frac{\mathrm{E}_{z} \mathrm{E}_{\bar{z}}}{|\mathrm{E}|^{2}+1} \overline{\mathrm{E}}, \quad \mathrm{p}_{z \bar{z}}=0
$$

Conversely, any solution of this Ernst-type equation defines uniquely via the generalized Lelieuvre formulae (6) a generalized Weingarten surface of Class 2.

It is evident that the Ernst-type equation (1) may be derived in a similar manner by considering generalized Weingarten surfaces in Minkowski space $\mathbb{M}^{3}$. The geometric results obtained in the following therefore hold mutatis mutandis for the Ernst-Weyl equation.

\section{Helicoids and the Painlevé $V$ equation}

In [6], it has been shown that the Ernst-type equation (9) admits special solutions in terms of particular Painlevé III transcendents which are associated with generalized Weingarten surfaces of revolution. It may be verified that the underlying particular Painlevé III equation constitutes a degenerate case of the Painlevé V equation. From a geometric point of view, this is seen as follows.

It is natural to regard helicoids as canonical generalizations of surfaces of revolution. These are generated by (twisted) curves which are simultaneously rotated about and translated along a fixed axis at constant speed [3]. Here we consider generators of the form $\Gamma: r=r(x, y=$ const), where the coordinates $x$ and $y$ are given by the decomposition $z=x+\mathrm{i} y$, so that the generalized Lelieuvre formulae (6) become

$$
r_{x}=\rho N_{y} \times N+\mu N_{x}, \quad r_{y}=\rho N \times N_{x}+\mu N_{y} .
$$

Thus, if we choose the parametrization

$$
\boldsymbol{r}=\left(\begin{array}{c}
F(x) \cos [y+G(x)] \\
F(x) \sin [y+G(x)] \\
c y+H(x)
\end{array}\right)
$$


of a helicoid $\Sigma$, where the constant $c$ is called the parameter of the helicoidal motion, then it is readily verified that the unit normal to $\Sigma$ assumes the form

$$
N=\left(\begin{array}{c}
\sin \varphi \cos \omega \\
\sin \varphi \sin \omega \\
\cos \varphi
\end{array}\right), \quad \varphi=\varphi(x), \quad \omega=y+\psi(x)
$$

It is noted that the case $c=0$ corresponds to surfaces of revolution.

It turns out that generalized Weingarten surfaces of Class 2 which admit spherical representations of the form (12) are not necessarily helicoidal. Accordingly, we relax the above restriction on $\Sigma$ and demand that the 'offset surface' $\Sigma^{o}$ defined by

$$
r^{o}=r-\mu N
$$

be helicoidal. If $\mu$ is constant then $\Sigma$ and $\Sigma^{\circ}$ constitute parallel surfaces. The generalized Lelieuvre formulae for the offset surface read

$$
r_{x}^{o}=\rho N_{y} \times N-\mu_{x} N, \quad r_{y}^{o}=\rho N \times N_{x}-\mu_{y} N
$$

where $N$ is not normal to the offset surface $\Sigma^{o}$ unless $\mu$ is constant. However, if we choose the harmonic functions $\rho$ and $\mu$ to be

$$
\rho=c_{0} x+c_{1}, \quad \mu=a x+b y+c_{2},
$$

with arbitrary constants $c_{i}$ and $a, b$, then the relations

$$
r_{x}^{o} \cdot N=-a, \quad r_{y}^{o} \cdot N=-b
$$

imply that if $\Sigma^{o}$ is helicoidal with an associated position vector $r^{o}$ of the form (11) then the unit normal $N$ is still given by (12) for appropriate functions $\varphi$ and $\psi$. Conversely, it is shown below that if the spherical representation of a generalized Weingarten surface $\Sigma$ of Class 2 is given by (12) then the corresponding offset surface $\Sigma^{\circ}$ is helicoidal.

Insertion of the parametrization (12) into the generalized Lelieuvre formulae (10) now produces the relations

$$
\begin{aligned}
& \boldsymbol{r}_{x}=\left(\rho \sin \varphi+\mu \varphi^{\prime}\right)\left(\begin{array}{c}
\cos \varphi \cos \omega \\
\cos \varphi \sin \omega \\
-\sin \varphi
\end{array}\right)+\mu \psi^{\prime} \sin \varphi\left(\begin{array}{c}
-\sin \omega \\
\cos \omega \\
0
\end{array}\right), \\
& r_{y}=\left(\rho \varphi^{\prime}+\mu \sin \varphi\right)\left(\begin{array}{c}
-\sin \omega \\
\cos \omega \\
0
\end{array}\right)-\rho \psi^{\prime} \sin \varphi\left(\begin{array}{c}
\cos \varphi \cos \omega \\
\cos \varphi \sin \omega \\
-\sin \varphi
\end{array}\right),
\end{aligned}
$$


where the prime denotes differentiation with respect to $x$, and cross-differentiation yields

$$
\begin{gathered}
\left(\rho \varphi^{\prime}\right)^{\prime}=\rho\left(1+\psi^{2}\right) \sin \varphi \cos \varphi+\left(b \psi^{\prime}-a\right) \sin \varphi \\
\left(\rho \psi^{\prime} \sin \varphi\right)^{\prime}+\rho \psi^{\prime} \varphi^{\prime} \cos \varphi+b \varphi^{\prime}=0 .
\end{gathered}
$$

The latter differential equation admits the first integral

$$
\psi^{\prime}=\frac{b \cos \varphi+c}{\rho \sin ^{2} \varphi}
$$

so that $(13)_{1}$ may be written as

$$
\left(\rho \varphi^{\prime}\right)^{\prime}=\rho \sin \varphi \cos \varphi-a \sin \varphi+\frac{\left(b^{2}+c^{2}\right) \cos \varphi+b c\left(1+\cos ^{2} \varphi\right)}{\rho \sin ^{3} \varphi} .
$$

Finally, the position vector of the generalized Weingarten surface $\Sigma$ is readily shown to be

$$
r=\left(\begin{array}{c}
f \cos \omega-g \sin \omega \\
f \sin \omega+g \cos \omega \\
c y+h
\end{array}\right)+\mu N,
$$

where the functions $f, g$ and $h$ are given by

$$
f=\rho \varphi^{\prime}, \quad g=\left(b+\rho \psi^{\prime} \cos \varphi\right) \sin \varphi, \quad h=-\int\left(\rho \sin ^{2} \varphi+a \cos \varphi\right) d x
$$

We therefore conclude that the offset surface $\Sigma^{o}$ is indeed helicoidal and the parameter $c$ of the helicoidal motion is identified as the constant of integration in the first integral (14).

The parametrization (16) shows that if $b=0$ then the surface $\Sigma$ is also helicoidal. If, in addition, $c=0$ then $\Sigma$ is a surface of revolution, in which case the differential equation (15) reduces to the stationary double sine-Gordon equation or a particular Painlevé III equation in trigonometric form depending on whether $\rho^{\prime}=0$ or $\rho^{\prime} \neq 0$ [6]. Here, we focus on the generic case $\rho^{\prime} \neq 0$ and therefore $\operatorname{set}^{1} \rho=x$ without loss of generality. Remarkably, in this case, the integral $(17)_{3}$ may be evaluated explicitly to obtain

$$
h=\frac{1}{2} x^{2}\left[\varphi^{2}+\left(\psi^{2}-1\right) \sin ^{2} \varphi\right]-a x \cos \varphi
$$

\footnotetext{
${ }^{1}$ In the context of general relativity, the case $\rho^{\prime}=0$ is non-physical.
} 
Now, on setting $\varphi=2 \arctan \sqrt{-w}$, the differential equation (15) reduces to the Painlevé $V$ equation

$$
w^{\prime \prime}=\left(\frac{1}{2 w}+\frac{1}{w-1}\right) w^{2}-\frac{w^{\prime}}{x}+\frac{(w-1)^{2}}{x^{2}}\left(\alpha w+\frac{\beta}{w}\right)+\frac{\gamma w}{x}+\frac{\delta w(w+1)}{w-1}
$$

with parameters $\alpha=-(b-c)^{2} / 8, \beta=(b+c)^{2} / 8, \gamma=-2 a, \delta=-2$. It is evident that the coordinate $x$ may be scaled in such a way that $\delta$ takes any negative value. Thus, up to complexification, the Painlevé $V$ equation with four arbitrary parameters has been derived in a purely geometric manner.

\section{Sphere congruences and a Bäcklund transformation}

Generalized Weingarten surfaces of Class 2 have been shown to come naturally in pairs [6]. Thus, if $\Sigma$ is a generalized Weingarten surface of Class 2 then one may associate with each point $P$ on $\Sigma$ a sphere of radius $\rho-\mu$ which touches ${ }^{2}$ $\Sigma$ at $P$ so that $\Sigma$ constitutes one sheet of the envelope of a two-parameter family of spheres (sphere congruence). The second sheet $\Sigma_{-}$turns out to be another generalized Weingarten surface of Class 2 with position vector

$$
r_{-}=r+(\rho-\mu)\left(N-N_{-}\right)
$$

In terms of the Ernst potential $E$, the unit normal $N_{-}$is given by

$$
E_{-}=\frac{E\left(|E|^{2}+1\right) p_{\bar{i}}+2 \Re(p) E_{\bar{z}}}{\left(|E|^{2}+1\right) p_{\bar{z}}-2 \Re(p) \bar{E}_{\bar{z}}}, \quad p_{-}=p+2 i v
$$

where the harmonic function $v$ is defined as in (3). The transformation (19) which, by construction, leaves invariant the Ernst-type equation (9) represents the analogue of the Laplace-Darboux-type transformation $\mathscr{L}_{-}$for the Ernst-Weyl equation (cf. (2)).

Inspection of the transformation formula (19) now shows that the above LaplaceDarboux-type transformation $\mathscr{L}_{-}$maps within the class of helicoidal offset surfaces $\Sigma^{o}$. Indeed, it is seen that the specialization $\mathrm{E}(x, y)=\mathrm{E}(x) e^{\mathrm{i} y}$, which corresponds to the ansatz (12), is preserved by $\mathscr{L}_{-}$. Specifically, comparison of the parametrizations (7) and (12) yields $\mathrm{E}(x)=e^{i \psi} \cot (\varphi / 2)$ so that evaluation of (19) $)_{1}$ results in the Bäcklund transformation

$$
w w_{-}=1+4(w-1)^{2} \frac{(a-1)\left[2 x w^{\prime}+4 x w+(a-1)\left(w^{2}-1\right)\right]+b c(w-1)^{2}}{4\left[x w^{\prime}+2 x w+(a-1)(w-1)\right]^{2}+(b-c)^{2}(w-1)^{4}}
$$

\footnotetext{
${ }^{2}$ If $\rho-\mu$ is positive then the centre of the sphere is assumed to be on the same side of $\Sigma$ as the unit normal $N$.
} 
for the Painlevé V equation (18). The remaining relation $(19)_{2}$ implies that $^{3}$

$$
\mu_{-}=\mu-2 \rho
$$

by virtue of (3) and (8). Moreover, it may be directly verified that the constant of integration in the first integral (14) remains unchanged. Thus, as a consequence of the transformation laws

$$
a_{-}=a-2, \quad b_{-}=b, \quad c_{-}=c,
$$

we obtain the new parameters

$$
\alpha_{-}=\alpha, \quad \beta_{-}=\beta, \quad \gamma_{-}=\gamma+4, \quad \delta_{-}=\delta .
$$

It would be of interest to investigate whether the Bäcklund transformation (20) may be decomposed into known elementary Bäcklund transformations for the Painlevé $\mathrm{V}$ equation [4].

\section{References}

[1] G. A. Alekseev, "Soliton configurations of interacting massless fields", Sov. Phys. Dokl. 28 (1983) 133-135.

[2] A. I. Bobenko, "Surfaces in terms of 2 by 2 matrices. Old and new integrable cases", in Harmonic Maps and Integrable Systems (eds. A. Fordy and J. Wood), (Vieweg, 1994) 83-128.

[3] L. P. Eisenhart, A Treatise on the Differential Geometry of Curves and Surfaces (Dover, New York, $1960)$.

[4] V. I. Gromak, "Bäcklund transformations of Painlevé equations and their applications", in The Painlevé Property. One Century Later (ed. R. Conte), (Springer, New York, 1999) 687-734.

[5] B. G. Konopelchenko and C. Rogers, "On 2+1-dimensional nonlinear systems of Loewner-type", Phys. Lett. A 158 (1991) 391-397.

[6] W. K. Schief, "On Laplace-Darboux-type sequences of generalized Weingarten surfaces", J. Math. Phys., 41 (2000) 855-870.

[7] W. K. Schief, "On a 2 + 1-dimensional integrable Ernst-type equation", Proc. R. Soc. London A 446 (1994) 381-398.

[8] W. K. Schief, "The Painlevé III, V and VI transcendents as solutions of the Einstein-Weyl equations", Phys. Lett. A 267 (2000) 265-275.

[9] W. K. Schief and C. Rogers, "On a Laplace sequence of nonlinear integrable Ernst-type equations", in Algebraic Aspects of Integrable Systems (eds. A. S. Fokas and I. M. Gelfand), (Birkhäuser, Boston, 1997) 315-321.

[10] N. R. Sibgatullin, "A proof of Geroch's hypothesis for electromagnetic and neutrino fields in the general theory of relativity", Sov. Phys. Dokl. 28 (1983) 552-554.

\footnotetext{
${ }^{3}$ It may be shown that the constant of integration has to vanish [6].
} 